\title{
Do personality traits predict post-traumatic stress?: a prospective study in civilians experiencing air attacks
}

\author{
GORAN KNEŽEVIĆC ${ }^{1,2}$, GORAN OPAČIĆ ${ }^{1,2}$, DANKA SAVIĆ ${ }^{1}$ AND STEFAN PRIEBE ${ }^{3 *}$ \\ ${ }^{1}$ Psychosocial Centre and Centre for Rehabilitation of Torture Victims, International Aid Network, Belgrade, \\ Serbia and Montenegro; ${ }^{2}$ Department of Psychology, University of Belgrade, Serbia and Montenegro; \\ ${ }^{3}$ Unit for Social and Community Psychiatry, Barts and the Royal London School of Medicine, \\ Queen Mary, University of London, UK
}

\begin{abstract}
Background. Previous studies have suggested an association between personality traits and posttraumatic stress. These studies either focused exclusively on military veterans or assessed personality traits after the traumatic event. This study investigates to what extent personality traits as assessed before the traumatic experience predict post-traumatic stress in civilians experiencing air attacks at the end of the exposure to stressful events and 1 year later.

Method. The revised version of the NEO Personality Inventory was administered to 70 students in Belgrade, Yugoslavia. In 1999, 1 or 2 years after the assessment, all students were exposed to air attacks for 11 weeks. At the end of the attacks and 1 year later post-traumatic stress was measured on the Impact of Event Scale.
\end{abstract}

Results. Pre-trauma personality predicted $13 \%$ of the variance of intrusion scores 1 year after the attacks. There was no significant correlation between personality traits and subsequent avoidance scores at any point of time.

Conclusions. Personality traits that are assessed before a traumatic event can, to a limited extent, predict intrusive symptoms in a non-clinical sample of civilians. Pre-trauma assessments of personality might be less strongly associated with post-traumatic stress than personality traits obtained after the traumatic event.

\section{INTRODUCTION}

In the search for factors that predict response to traumatic events, various studies have investigated the relationship between personality traits and post-traumatic stress. They suggest that personality traits influence the response to traumatic events and predict the level of posttraumatic stress (e.g. Sutker et al. 1991, 1995; Bramsen et al. 2000; Lecic-Tosevski et al. 2003). More post-traumatic stress symptoms have been found in patients with higher scores of neuroticism - alone (Breslau et al. 1991; Hyer

* Address for correspondence: Professor Stefan Priebe, Unit for Social \& Community Psychiatry, Academic Unit, Newham Centre for Mental Health, London E13 8SP, UK.

(Email: s.priebe@qmul.ac.uk) et al. 1994) or in combination with introversion (Davidson et al. 1987; McFarlane, 1988b; Fauerbach et al. 2000)-and negativism (McFarlane, 1988a), and with low agreeableness (Talbert et al. 1993). The findings on which personality factors are associated with posttraumatic stress have not been entirely consistent, which may be due to differences in the instruments used to assess personality. The amount of variance of post-traumatic stress explained by personality factors has been low to moderate.

Most studies in the field have serious methodological shortcomings, most notably that personality was assessed after the event when ratings may be influenced by psychological sequelae of the traumatic experience. One 
exception is a study on the sequelae of World War II combat (Lee et al. 1995), in which, however, personality was not directly measured with established and validated methods. Three other studies evaluated personality tests at the age of 15 years, entrance to undergraduate school or at selection for the Army, and linked the results to symptoms of post-traumatic stress after combat or peacekeeping missions respectively. In these studies, the interval between personality assessment and traumatic events varied, as did the time between combat experience and assessment of post-traumatic stress. The interval between assessment of personality and posttraumatic stress ranged between several years and more than two decades. Schnurr et al. (1993) and Bramsen et al. (2000) reported an association between personality - as assessed on the Minnesota Multiphasic Personality Inventory - and post-traumatic stress symptoms, whilst Card (1983) did not find any predictive value of personality traits for post-traumatic stress many years later. The impact of some personality traits on post-traumatic stress held true when the influence of exposure to traumatic events was controlled for.

This study investigated the impact of personality factors on post-traumatic stress in civilians who experienced air attacks in Belgrade, Yugoslavia. The attacks lasted for 78 days in spring 1999 and resulted in civilian casualties. In contrast to the aforementioned prospective studies, personality was assessed just 1 or 2 years before the events, all subjects were exposed to the same air attacks over a confined period of time, and post-traumatic stress was consistently assessed at the end of exposure and 1 year later.

\section{METHOD}

All subjects were psychology students at the University of Belgrade. They had filled in the NEO Personality Inventory (NEO-PI; Costa $\&$ McCrae, 1992) as part of a course in their second year, 1 or 2 years before spring 1999. Seventy students were contacted at the end of the air attacks in June 1999 (62 women, 8 men; age: mean $=22.4$ years, s.D. $=1 \cdot 6$ ) and rated post-traumatic stress on the Impact of Event Scale (IES; Horowitz et al. 1979; Sundin \& Horowitz, 2002). At that time they also filled in a 37-item checklist of stressful experiences relating to the air attacks that reflects the nature and degree of personal exposure to stressful events such as witnessing the death of and injuries to nearby persons, being close to powerful explosions or being in a bombed building (Gavrilovic et al. 2002). Fifty-four students rerated the IES 1 year later. Written informed consent was obtained from all participants.

Pearson's correlation coefficients were calculated to test the association of personality traits and degree of exposure to stressful events with scores of intrusion and avoidance at the end of the attacks and 1 year later. The five pre-trauma personality traits were entered blockwise as predictors in multiple regression analyses with intrusion and avoidance scores as dependent variables. In a second step, the degree of exposure was also entered as a predictor to analyse the combined predictive power of personality traits and degree of exposure, and to test whether personality factors interact with degree of exposure in predicting post-traumatic stress.

\section{RESULTS}

Mean scores and standard deviations on NEOPI were 78.8 (s.D. $=21 \cdot 7$ ) for neuroticism, $117 \cdot 8$ $($ S.D. $=18 \cdot 1)$ for extraversion, $132 \cdot 8$ (s.D. $=16 \cdot 2)$ for openness, $115 \cdot 2$ (s.D. $=16 \cdot 2$ ) for agreeableness and $118 \cdot 8$ (s.D. $=20 \cdot 2$ ) for conscientiousness. Intrusion scores on IES at the end of the attacks were 11.04 (s.D. $=7 \cdot 15$ ) and 4.67 (s.D. $=$ $5 \cdot 56) 1$ year later $(t$ test for dependent samples; $t=6.57, \mathrm{df}=53, p<0.001)$. Avoidance scores were 11.96 (S.D. $=6.99)$ at the end of attacks and 6.38 (s.D. $=6.06) 1$ year afterwards $(t=4 \cdot 81$, $\mathrm{df}=53, p<0 \cdot 001)$. Intrusion scores at the two points were significantly correlated $(r=0 \cdot 39, p=$ $0 \cdot 003)$, whilst avoidance scores were not $(r=$ $0 \cdot 19, p=0 \cdot 187)$. At the end of the attacks, 10 out of the 54 students who were interviewed twice had an IES total score of $>34$, which has been suggested as a threshold for clinically relevant symptoms (Neal et al. 1994) equivalent to a diagnosis of Post-traumatic Stress Disorder, although a diagnosis cannot be made on the basis of the IES alone. After 1 year, only two students had total IES scores above that threshold.

Table 1 shows how pre-trauma personality traits and degree of exposure to stressful events during attacks were associated with intrusion 
Table 1. Associations of pre-trauma personality traits as assessed on the NEO-PI and degree of exposure to stressful events during air attacks with IES scores for intrusion and avoidance at the end of attacks $(n=70)$ and 1 year later $(n=54)$ (Pearsons's $r$ and two-tailed $p$ values)

\begin{tabular}{ccccccc}
\hline \hline & & & & & & $\begin{array}{c}\text { Degree of } \\
\text { exposure }\end{array}$ \\
& Neuroticism & Extraversion & Openness & Agreeableness & Conscientiousness \\
\hline Intrusion at & $0 \cdot 23$ & $-0 \cdot 12$ & $0 \cdot 19$ & $0 \cdot 08$ & $-0 \cdot 07$ & $0 \cdot 20$ \\
the end of attacks & $(0 \cdot 057)$ & $(0 \cdot 338)$ & $(0 \cdot 123)$ & $(0 \cdot 499)$ & $(0 \cdot 565)$ & $(0 \cdot 094)$ \\
Avoidance at the & $0 \cdot 15$ & $-0 \cdot 10$ & $-0 \cdot 04$ & $-0 \cdot 10$ & $0 \cdot 03$ & $0 \cdot 12$ \\
end of attacks & $(0 \cdot 206)$ & $(0 \cdot 401)$ & $(0 \cdot 753)$ & $(0 \cdot 403)$ & $(0 \cdot 815)$ & $(0 \cdot 305)$ \\
Intrusion after & $0 \cdot 20$ & $-0 \cdot 06$ & $0 \cdot 30$ & $0 \cdot 14$ & $-0 \cdot 04$ & $0 \cdot 16$ \\
1 year & $(0 \cdot 072)$ & $(0 \cdot 331)$ & $(0 \cdot 014)$ & $(0 \cdot 151)$ & $(0 \cdot 393)$ & $(0 \cdot 124)$ \\
Avoidance after & $0 \cdot 18$ & $-0 \cdot 06$ & $0 \cdot 11$ & $-0 \cdot 03$ & $0 \cdot 17$ & $-0 \cdot 03$ \\
1 year & $(0 \cdot 193)$ & $(0 \cdot 680)$ & $(0 \cdot 448)$ & $(0 \cdot 859)$ & $(0 \cdot 221)$ & $(0 \cdot 843)$ \\
\hline \hline
\end{tabular}

and avoidance scores at the end of attacks and 1 year later.

A higher degree of exposure to stressful events during the attacks tended to be correlated with higher intrusion scores at the end of attacks, but this correlation failed to reach statistical significance $(r=0 \cdot 20, p=0 \cdot 094)$. Degree of exposure did not significantly predict intrusion after 1 year or avoidance at any point of time. With respect to personality traits, higher scores of neuroticism were associated with more intrusion symptoms at the end of attacks and 1 year later with the correlation coefficients just failing to reach statistical significance. Openness was moderately correlated with intrusion after 1 year. Other correlations were not significant.

In multiple regression analyses with all five personality traits and degree of exposure as predictors, there was no significant prediction of intrusion scores at the end of air attacks, or of avoidance scores at any time. Yet, when all personality traits were considered as predictors, intrusion scores 1 year after the air attacks were significantly predicted. The explained variance was $13 \% \quad\left(R=0 \cdot 45, R^{2}=0 \cdot 21\right.$, adjusted $R^{2}=$ $0 \cdot 13 ; F=2 \cdot 483, \mathrm{df}=5,48, p=0 \cdot 044)$. In the regression function openness was identified as a significant predictor $[\beta=0.39, t(48)=2.833$, $p<0.001]$, whilst the predictive value of neuroticism failed to reach statistical significance $[\beta=0 \cdot 28, t(48)=1 \cdot 885, p=0 \cdot 06]$. The additional inclusion of the degree of exposure to stressful events as a potential predictor did not increase the amount of explained variance in intrusion scores 1 year after air attacks $\left[R=0.46, R^{2}=\right.$ $0 \cdot 21$, adjusted $R^{2}=0 \cdot 11 ; \quad F=2 \cdot 137, \quad \mathrm{df}=6$, $47, p=0 \cdot 066$; degree of exposure $\beta=0 \cdot 10$, $t(47)=0 \cdot 729, p=0 \cdot 47]$. There were no significant interaction effects between personality factors and degree of exposure.

\section{DISCUSSION}

To our knowledge, this is the first prospective study to investigate the impact of personality traits on post-traumatic stress that used the NEO-PI to assess personality traits, which may be regarded as one of the best-established instruments for that purpose at present. The study is also unusual in other respects: as a prospective study it is the only one that $(a)$ investigated civilians and not military veterans, $(b)$ had a mixed gender sample - in fact a predominantly female one, $(c)$ assessed personality traits and post-traumatic stress at fixed points of time in relation to the stressful experience and a short period of time before and after it, and $(d)$ obtained post-traumatic stress scores at two points of time, i.e. immediately after the end of exposure and 1 year later. In line with recommendations by Schnurr \& Vielhauer (2000) the study followed 'the ideal non-experimental design ... [with] multivariate statistical procedures, pre-traumatic personality measures and multiple posttraumatic assessments'. Moreover, the sample was homogeneous with respect to age, educational background, current occupation as well as the nature and duration of the stressful events. Thus, these potentially influential factors cannot have substantially confounded the findings.

Methodological weaknesses of the study are the small size and selective nature of the sample. The small sample size was due to the inevitably 
opportunistic nature of the study (exposure to traumatic events is difficult to anticipate in civilians) and has clearly limited the statistical power of the analysis.

When post-traumatic stress was assessed directly at the end of the 78-day period of attacks, acute stress reactions and post-traumatic stress may have overlapped in influencing IES ratings. This overlap at the end of the attacks and a favourable spontaneous course of post-traumatic stress are likely to have contributed to the significant decrease of intrusion and avoidance scores between the end of the attacks and the 1-year follow-up.

The only other significant result - in univariate and multivariate analyses - was that openness was a predictor of intrusion after 1 year. The predictive value of openness has so far not been reported in other studies. A higher degree of openness might increase the vulnerability to develop stress symptoms following a traumatic event. It might also interfere with denial as a successful coping mechanism, both processes resulting in a higher level of intrusion symptoms.

Supposing that openness might decrease as a result of post-traumatic stress, the association found in this prospective study would not necessarily be identified in cross-sectional studies after the trauma. Also, the level of openness in the sample of this study was relatively high (Card, 1983). The impact of openness on developing post-traumatic stress might - following a nonlinear association-be stronger at higher levels.

In general, the predictive power of personality variables in this study appears low. Avoidance scores were not predicted at all, and the variance of intrusion scores explained through personality traits did not exceed $13 \%$. Most tested correlations were not significant, and adjustment for multiple testing of correlations would have made all significant results disappear. Even taking into account the limited statistical power of the study, the findings suggest that personality traits - although of importance - are not the main determinants of post-traumatic stress response. They appear to predict intrusion rather than avoidance. In this study, avoidance scores were less stable over time than intrusion, which might indicate a low reliability of the rating. A low reliability of the dependent variable, i.e. avoidance, makes it more difficult to identify significant predictors. It remains unclear to what extent the results are specific to the sample and the nature of the potentially traumatic events. The level of exposure to stressful events was measured, but is difficult to compare with levels of exposure to other types of events of samples in other studies. The level of stressful events did not predict post-traumatic stress, possibly because the nature and degree of exposure was relatively similar across the sample. Moreover, there was no significant interaction between personality traits and degree of exposure, as has been found in a study that investigated a similar sample with a retrospective design (Lecic-Tosevski et al. 2003).

The amount of explained variance is similar to the findings of Bramsen et al. (2000) in a much larger sample. Some cross-sectional studies with post-trauma assessments of personality suggest a much stronger association between personality and post-traumatic stress than found in the few prospective investigations (Lauterbach \& Vrana, 2001). One may speculate as to whether studies with post-trauma personality assessments tend to overestimate the association between personality traits and post-traumatic stress because of post-traumatic personality changes or biases influencing all ratings done at the same time. This possibility should be considered in future studies on factors determining post-traumatic stress, many of which will - for various practical and ethical reasons - not be in a position to use pre-trauma assessments of personality.

\section{DECLARATION OF INTEREST}

None.

\section{REFERENCES}

Bramsen, I., Dirkzwager, A. J. \& van der Ploeg, H. M. (2000). Predeployment personality traits and exposure to trauma as predictors of post-traumatic stress syndrome: a prospective study of former peacekeepers. American Journal of Psychiatry 157 1115-1119.

Breslau, N., David, G. C., Andreski, P. \& Peterson, E. (1991) Traumatic events and post-traumatic stress disorder in an urban population of young adults. Archives of General Psychiatry 48, 216-222.

Card, J. J. (1983). Lives after Vietnam: The Personal Impact of Military Service. Lexington Books: Lexington, MA.

Costa, P. T. \& McCrae, R. R. (1992). The Revised NEO Personality Inventory Manual. Psychological Assessment Resources: Odessa, FL. 
Davidson, J., Kudler, H. \& Smith, R. (1987). Personality in chronic post-traumatic stress disorder: a study of the Eysenck inventory. Journal of Anxiety Disorders 1, 295-300.

Fauerbach, J. A., Lawrence, J. W., Schmidt, C. W., Munster, A. M. \& Costa, P. T. (2000). Personality predictors of injury-related posttraumatic stress disorder. Journal of Nervous and Mental Diseases 188, 510-517.

Gavrilovic, J., Lecic-Tosevski, D., Knezevic, G. \& Priebe, S. (2002). Predictors of post-traumatic stress in civilians-a study of Yugoslavian students. Journal of Nervous and Mental Diseases 190, 257-262.

Horowitz, M. J., Wilner, N. \& Alvarez, W. (1979). Impact of event scale: a measure of subjective stress. Psychosomatic Medicine 41, 209-218.

Hyer, L., Braswell, L., Albrecht, B., Boyd, S., Boudewyns, P. \& Talbert, S. (1994). Relationship of NEO-PI to personality styles and severity of trauma in chronic PTSD victims. Journal of Clinical Psychology 50, 699-707.

Lauterbach, D. \& Vrana, S. (2001). The relationship among personality variables, exposure to traumatic events and severity of post-traumatic stress syndromes. Journal of Traumatic Stress 14, $29-45$.

Lecic-Tosevski, D., Gavrilovic, J., Knesevic, K. \& Priebe, S. (2003). Personality factors and posttraumatic stress: associations in civilians one year after air attacks. Journal of Personality Disorders $\mathbf{1 7}$ 537-549.

Lee, K. A., Vaillant, G. E., Torrey, W. C. \& Elder, G. H. (1995). A 50 -year prospective study of the psychological sequelae of World War II combat. American Journal of Psychiatry 152, 516-522.
McFarlane, A. C. (1988a). The longitudinal course of post-traumatic morbidity: The range of outcomes and their predictors. Journal of Nervous and Mental Diseases 176, 30-39.

McFarlane, A. C. $(1988 b)$. The aetiology of post-traumatic stress disorders following a natural disaster. British Journal of Psychiatry 152, 116-121.

Neal, A. L., Busuttil, W., Rollins, J. W., Herepath, R., Strike, P. \& Turnbull, G. J. (1994). Convergent validity of measures of post-traumatic stress disorder in a mixed military and civilian population. Journal of Traumatic Stress 7, 447-455.

Schnurr, P. P., Friedman, M. J. \& Rosenberg, S. D. (1993) Preliminary MMPI scores as predictors of combat-related PTSD symptoms. American Journal of Psychiatry 150, 479-483.

Schnurr, P. P. \& Vielhauer, M. J. (2000). Personality as a risk factor for PTSD. In Risk Factors for Post-traumatic Stress Disorder (ed. R. Yehuda), pp. 191-222. American Psychiatric Press: Washington, DC.

Sundin, E. C. \& Horowitz, M. J. (2002). Impact of Event Scale: psychometric properties. British Journal of Psychiatry 180, 205-209.

Sutker, P. B., Bugg, F. \& Allain, A. N. (1991). Psychometric prediction of PTSD among POW survivors. Psychological Assessment $\mathbf{3}$ $105-110$.

Sutker, P. B., Davis, J. M., Uddo, M. \& Ditta, S. R. (1995). War zone stress, personal resources and PYSD in Persian Gulf War returnees. Journal of Abnormal Psychology 104, 444-452.

Talbert, F. S., Braswell, L. C., Albrecht, J. W., Hyer, L. A. \& Boudewyns, P. A. (1993). NEO-PI profiles PTSD as a function of trauma level. Journal of Clinical Psychology 49, 663-669. 